\title{
Investigation of the potential mechanism of the Shugan Xiaozhi decoction for the treatment of nonalcoholic fatty liver disease based on network pharmacology and molecular docking
}

Yufeng Xing ( $\nabla$ yufeng000729@163.com )

Shenzhen Traditional Chinese Medicine Hospital https://orcid.org/0000-0001-6762-3788

Rong Yang

Macau University of Science and Technology

Huili Yang

Guangzhou University of Chinese Medicine

Dansheng Jiang

Guangzhou University of Chinese Medicine

Linyi Xu

Guangzhou University of Chinese Medicine

Lian Feng

Guangzhou University of Chinese Medicine

\section{Research}

Keywords: Shugan Xiaozhi decoction, Nonalcoholic fatty liver disease (NAFLD), Network pharmacology, Molecular docking, Inflammation

Posted Date: February 2nd, 2022

DOI: https://doi.org/10.21203/rs.3.rs-1294807/v1

License: (c) (i) This work is licensed under a Creative Commons Attribution 4.0 International License. Read Full License 


\section{Abstract}

Background: Nonalcoholic fatty liver disease (NAFLD) is a metabolic-related disease with its incidence increasing annually. Shugan Xiaozhi (SGXZ) decoction, a composite traditional Chinese medicinal prescription, was demonstrated to exert a therapeutic effect on NAFLD. However, the underlying mechanisms were incompletely elucidated. In this study, the potential bioactive ingredients and mechanism of the SGXZ decoction against NAFLD were explored via network pharmacology and molecular docking.

Methods: Compounds in SGXZ decoction were identified and collected from literature studies, and the corresponding targets were predicted through SEA database. Potential targets related to NAFLD were overlapped by using DisGeNET and GeneCards database. The compound-target-disease and PPI network were then constructed based on the putative intersection targets of SGXZ decoction and NAFLD to recognize the key compounds and targets. Functional enrichment analysis was performed to elucidate the biological process and signaling pathway that SGXZ decoction treated NAFLD. Finally, molecular docking combined with homology modeling was applied to further verify the binding between key active compounds and targets.

Results: A total of 31 active compounds and 220 corresponding targets in SGXZ decoction were collected. Moreover, 1544 overlapped targets of NAFLD were obtained, of which 78 common targets were intersected with targets of SGXZ decoction. Key compounds and targets were recognized from compound-target-disease and PPI network. Functional enrichment analysis revealed that multiple biological pathways were annotated including insulin resistance, HIF-1, PI3K-Akt, and MAPK signaling pathways. Molecular docking confirmed that gallic acid, chlorogenic acid and isochlorogenic acid A could combine firmly with all of the key targets.

Conclusion: SGXZ decoction could produce a promising effect for its multi-component, multi-target and multi-biological process in the treatment of NAFLD. The present study provides the novel insight into a comprehensive understanding for further study.

\section{Introduction}

Nonalcoholic fatty liver disease (NAFLD) is a progressive condition ranging from simple NAFL to nonalcoholic steatohepatitis (NASH), liver fibrosis, liver cirrhosis and even worse, the hepatocellular carcinoma (HCC) [1-2]. It is a metabolic disease representing the hepatic manifestation of a systemic metabolic disorder [3], and is demonstrated to be associated with obesity-related disorders and diabetes [4-5]. With the increase epidemic of obesity and metabolic-related comorbidities, global incidence of NAFLD is estimated to be $25 \%$ and keeps rising continually [6]. NAFLD has been one of the most leading courses of chronic hepatic disease, affecting approximately 1.7 billion individuals worldwide [7]. Moreover, it is one of the most common indication for liver transplantation and NAFLD-HCC is now the fastest growing demand of liver transplantation in the USA [8-9]. Lifestyle modification including healthy 
diet and increased physical activity is recommended as the first-line treatment in NAFLD management [10]. Nevertheless, their effectiveness is limited in NAFLD patients due to the low readiness to change lifestyle, especially in terms of increasing physical activity [11]. Another challenge for the lifestyle intervention has been the occurrence of weight regain [12-13]. Up to date, there are no FDA-approved pharma therapies for the treatment of NAFLD at present [14]. Insulin sensitizer (rosiglitazone, pioglitazone and metformin), antioxidants (vitamin E), anti-inflammatory and lipid lowering drugs (atorvastatin and simvastatin) have been in practice for the treatment of NAFLD [15-16]. However, usage of rosiglitazone could increase the risk of cardiovascular causes [17], while long-term use of vitamin $E$ [18-19] was reported to increase the risk of prostate cancer and might increase all-cause mortality. Moreover, the adverse outcome of pioglitazone [20-21], atorvastatin [22] and simvastatin [23] including weight gain, edema, heart failure, cytotoxicity and hepatotoxicity, should not be neglected. Therefore, it is an urgent need to develop pharmacological strategies for the treatment of NAFLD.

The complicated pathophysiological mechanism of NAFLD restricts the efficacy of the application of a single agent. Traditional Chinese Medicine (TCM) has been commonly used to treat hepatic disease and metabolic disorders-related obesity and type 2 diabetes mellitus [24-26]. The therapeutic effect of TCM on NAFLD mainly focused on the holistic regulations including lipid metabolism modulation, antioxidative stress, anti-inflammation, and gut microbiota modulation [27-29]. Shugan Xiaozhi (SGXZ) decoction is a composite traditional Chinese prescription composed of twelve herbs including pleuri Radix, Paeoniae radix alba (stir-baked), Aurantii Fructus Immaturus, Glycyrrhizae Radixet Rhizoma, Artemisiae Scopariae Herba, Gardeniae Fructus, Poria, Alismatis Rhizoma, Crataegi Fructus, Cassiae Semen, Nelumbinis Folium, and Pumex in a ratio of $2: 1: 3: 1: 6: 2: 4: 6: 6: 6: 6: 6$. Previous clinical studies demonstrated that the treatment of SGXZ could partially protected and restored the liver functions in NASH patients, which was associated with significant reduction of aminotransferases, total cholesterol and triacylglycerol, and repairment of intestinal mucosal barrier [30-31]. Consistently, researches indicated that SGXZ decoction exerted hepatoprotective effect through regulating fatty acid $\beta$ oxidation, relieving intestinal microecological disorder and repairing intestinal mucosal barrier, thereby ameliorating NAFLD in rat model [32-33]. However, therapeutic mechanisms of SGXZ decoction in the treatment of NAFLD have not been fully clarified, and the active ingredients and key targets of SGXZ remain to be further investigated.

Based on the integration of system biology, bioinformatic and pharmacology [34-36], network pharmacology is currently used to explore the potential pharmacological effect and underlying mechanisms of a drug on a disease [37-38]. In particular, it concentrates on the elucidation of complex biological relationship among the drugs, targets, pathways and diseases from a systemic and holistic perspective [39]. The wholeness, relevance and systematic nature of network pharmacology are in line with the concept and treatment theory of TCM, providing a novel method and powerful tool to decipher the therapeutic mechanisms of TCM [40]. In addition, molecular docking is a computational method based on the analysis of the binding pose and affinity between small molecule and macromolecular target, which is widely utilized to predict and identify the potentially active compounds [41-42]. In our study, network pharmacology combined with molecular docking was applied to explore the 
pharmacological and molecular mechanisms involved in the treatment of SGXZ decoction on NAFLD. First, chemical compounds in SGXZ decoction were collected and recognized through literature study and their corresponding targets were then obtained from Similarity ensemble approach (SEA) database. Second, the potential targets involved in the pathology of NAFLD were predicted from DisGeNET and GeneCards, and compared with the predicted targets of SGXZ decoction to intersect and identify the common targets. Subsequently, the network of "compound-target-disease" and protein-protein interaction (PPI) network were constructed to identify the potentially active ingredients and key targets. Furthermore, functional enrichment including Gene ontology (GO) and Kyoto Encyclopedia of Genes and Genomes (KEGG) was analyzed via DAVID to explore the potential mechanism of SGXZ decoction for the treatment of NAFLD. Last, active ingredients were screened against the key targets by performing molecular docking to identify the compounds treating NAFLD. The overall flowchart of this study is described in Figure 1.

\section{Materials And Methods}

\subsection{Collection of compounds and the putative targets of SGXZ decoction}

The active compounds in SGXZ decoction were identified and collected through literature studies. Then, 2D or 3D conformations of the compounds were downloaded either from the PubChem database (https://pubchem.ncbi.nlm.nih.gov/) or through sketching in ChemDraw software. Moreover, the simplified molecular input line entry system (SMILS) numbers (Supplementary Table 1) of them were also acquired from PubChem or the conversion by ChemDraw. To predict the putative gene target of SGXZ decoction, the Simplified molecular input line entry system (SMILES) of each chemical compound was submitted into Similarity ensemble approach (SEA) database (https://sea.bkslab.org/), which relates proteins based on the set-wise chemical similarity among their ligands [43]. The predicted protein targets of SGXZ decoction were listed in Supplementary File 1.

\subsection{Identification of potential targets of NAFLD}

The "Non-alcohol Fatty Liver Disease" or the "Non-alcoholic Fatty Liver Disease" was used as the key words to predict the potential NAFLD-related gene targets by means of GeneCards (https://www.genecards.org/) and DisGeNET (https://www. disgenet.org/home/) disease database. DisGeNET is a discovery platform containing one of the largest publicly available collections of genes associated to human diseases [44]. Based on all annotated and predicted human genes, GeneCards provides comprehensive, user-friendly information via searchable, integrative database [45]. After removing the repetitive targets from the two databases, the NAFLD-related protein targets were retained for further study and the detailed information of the targets were provided in Supplementary File 2. 


\subsection{Construction of compound-target-disease network}

The SGXZ decoction-target network was first constructed based on the compounds and their predicted targets of SGXZ using Cytoscape software (Version 3.7.1). Venny diagram 2.1 version (https://bioinfogp.cnb.csic.es/tools/venny/) was used to intersect the protein targets between predicted targets of SGXZ decoction and potential targets of NAFLD. Then, the compound-target-disease network was established based on the intersected common targets and their corresponding compounds. Moreover, the degree value of nodes in compound-target-disease network was analyzed with NetworkAnalyzer plug-in in Cytoscape software. Degree indicates the total number of nodes connected to this node in the network, and nodes whose degree value were higher played more essential regulatory role in the network. The compounds and targets that contributed significantly to the construction of compound-target-disease network were recognized and selected as key active compounds and targets according to their higher degree value compared to the average degree value.

\subsection{Construction of protein-protein interaction (PPI) network}

PPI network can elucidate the interaction relationship between targets, which further helps to identify the key nodes and targets in the network. The intersected common targets were submitted to STRING database (https://string-db.org/,ver. 11.5) [46]. The species was limited to "homo sapiens" and the confidence of interaction score was set $\geq 0.400$ as default, which ensured a reasonable interaction between targets. Results with tabular text output (TSV) format of PPI network in SRTING were exported and then better visualized through Cytoscape software. The nodes with highest degree value were considered as the key targets which mediated the interaction of the network and participated in the treatment of SGXZ decoction on NAFLD.

\subsection{Enrichment analysis}

Functional annotation of the common targets in PPI network was analyzed through "Database for Annotation, Visualization and Integrated Discovery" (DAVID) database (https: //david.ncifcrf.gov/) to identify enriched biological themes of Gene ontology (GO) and visualize genes on Kyoto Encyclopedia of Genes and Genomes (KEGG) pathway maps. DAVID can provide a comprehensive set of functional annotation tools for investigators to understand biological meaning behind large list of genes and is widely used in bioinformatics [47-48]. Gene list of the common target proteins was uploaded into DAVID and the species was limited to "homo sapiens". The functional annotation of biological processes (BPs), molecular functions (MCs), cellular components (CCs) and KEGG pathways with $P$ value $\mathbb{0} 0.05$ were then performed for further analysis. Accordingly, target-pathway network was subsequently constructed using Cytoscape software. 


\subsection{Homology modeling and molecular docking}

3D conformational SDF format of the selected key chemical compounds were downloaded from PubChem. The crystal structures of the key protein targets were retrieved in RCSB Protein Data Bank database (PDB, https://www.rcsb.org/). For targets without released crystal structures, homology modeling was utilized to establish the structure based on the protein template. The construction was completed in SWISS-MODEL website (https://swissmodel.expasy.org/), which is a fully automated protein structure homology-modelling server [49]. Then, the quality of model structures was evaluated via "Ramachandran plot" in SAVES v6.0 website (https://saves.mbi. ucla.edu/). The target protein with neither crystal structure nor the template was excluded in molecular docking. Before docking, the compounds were charged with CHARMm force filed and minimized for optimization in Discovery Studio 2016 (DS) software. The target proteins were also loaded in DS, and waters and other redundant chains were firstly deleted. Then excess structures of the protein were removed, incomplete amino acid residues were supplemented, and hydrogen were added to optimize the conformation. CDOCKER module in DS was applied to calculate the binding energy between key active ingredients and key target proteins. CDOCKER is a grid-based molecular docking method on the basis of CHARMm force field, which can produce high-precision docking results. For docking parameters of CDOCKER, the size of docking box was set to $20 \AA \times 20 \AA \times 20 \AA$, "Top Hits" was set to 10 and "Pose Cluster Radius" was set to 0.5 . Moreover, the docking site of each target protein was obtained from previous research or the active cavities detected in DS (Supplementary Table 2). Only one top hit with best docking pose of each complex was reported and saved for further analysis.

\section{Results}

\subsection{Active compounds of SGXZ decoction and the corresponding targets}

31 active compounds of SGXZ decoction (SX01 SX31) were identified and collected from the previous studies with 2D chemical structures displayed in Fig. 2. Totally, 220 targets of SGXZ decoction were screened after removing duplicates.

\subsection{Potential targets of NAFLD and the compound-target- disease interaction}

Potential targets of non-alcohol Fatty Liver Disease (NAFLD) were independently screened in DisGeNET and GeneCards database. After the overlapped targets were deleted, 1544 potential protein targets of NAFLD from the two databases were retained. Venny diagram showed that 78 common targets between SGXZ and NAFLD related targets were intersected, which represented the potential targets of SGXZ in the treatment of NAFLD (Fig. 3A). 
The network of SGXZ decoction with its compounds and corresponding targets was first constructed to reveal the multi-target effect of SGXZ decoction (Fig. 3B). The network comprised of 252 nodes and 686 edges with 31 compounds and 220 corresponding targets. To further exhibited the directly regulatory effect of SGXZ decoction treating NAFLD, the network of compounds with common intersection targets was constructed (Fig. 3C), which consists of 109 nodes (31 compounds and 78 common targets) and 232 edges, suggesting a multi-component involved multi-targets of SGXZ decoction in the treatment of NAFLD. In addition, the top 10 compounds and common protein targets were obtained (Fig. 3D), of which the detailed information were listed in Table 1 and 2. Gallic acid possessed the highest degree value (degree=20), followed by kaempferol-3-0-galactoside (degree=19), licoricone (degree=17), isochlorogenic acid $A$ (degree=16), chlorogenic acid (degree=12), liquiritin (degree=12), gardenoside (degree=11), naringin (degree $=10)$, neohesperidin (degree $=10$ ) and poncirin (degree $=9$ ). These active compounds were recognized in SGXZ decoction to exert the pharmacologic effect on NAFLD. Moreover, as listed in Table 2, the top 10 targets with highest degree value included FGF1 (degree=15), VEGFA (degree=15), IL-2 (degree=13), LGALS3 (degree=11), SLC5A1 (degree=11), F3 (degree=10), ALDH1B1 (degree=7), CES2 (degree=7), IL-6 (degree=7) and P4HB (degree=7), which could be potentially acted on by the 10 key active compounds in SGXZ decoction.

Table 1

Key active compounds of SGXZ decoction in treatment of NAFLD

\begin{tabular}{|llllc|}
\hline Number & Ingredient & $\begin{array}{l}\text { Molecular } \\
\text { Formula }\end{array}$ & Pubchem_CID & Degree \\
\hline SX01 & Gallic acid & $\mathrm{C}_{7} \mathrm{H}_{6} \mathrm{O}_{5}$ & 370 & 20 \\
\hline SX02 & Naringin & $\mathrm{C}_{27} \mathrm{H}_{32} \mathrm{O}_{14}$ & 442428 & 10 \\
\hline SX03 & Neohesperidin & $\mathrm{C}_{28} \mathrm{H}_{34} \mathrm{O}_{15}$ & 442439 & 10 \\
\hline SX04 & Poncirin & $\mathrm{C}_{28} \mathrm{H}_{34} \mathrm{O}_{14}$ & 442456 & 9 \\
\hline SX05 & Gardenoside & $\mathrm{C}_{17} \mathrm{H}_{24} \mathrm{O}_{11}$ & 24721095 & 11 \\
\hline SX07 & Chlorogenic acid & $\mathrm{C}_{16} \mathrm{H}_{18} \mathrm{O}_{9}$ & 1794427 & 12 \\
\hline SX11 & Liquiritin & $\mathrm{C}_{21} \mathrm{H}_{22} \mathrm{O}_{9}$ & 503737 & 12 \\
\hline SX13 & Isochlorogenic acid A & $\mathrm{C}_{25} \mathrm{H}_{24} \mathrm{O}_{12}$ & 6474310 & 16 \\
\hline SX27 & Kaempferol-3-0-galactoside & $\mathrm{C}_{21} \mathrm{H}_{20} \mathrm{O}_{11}$ & 5282149 & 19 \\
\hline SX31 & Licoricone & $\mathrm{C}_{22} \mathrm{H}_{22} \mathrm{O}_{6}$ & 5319013 & 17 \\
\hline
\end{tabular}


Table 2

Key targets of SGXZ decoction in the treatment of NAFLD

\begin{tabular}{|llll|}
\hline UniprotKB & Target & Protein name & Degree \\
\hline P05230 & FGF1 & Fibroblast growth factor 1 & 15 \\
\hline P15692 & VEGFA & Vascular endothelial growth factor A & 15 \\
\hline P60568 & IL-2 & Interleukin-2 & 13 \\
\hline P17931 & LGALS3 & Galectin-3 & 11 \\
\hline P13866 & SLC5A1 & Sodium/glucose cotransporter 1 & 11 \\
\hline P13726 & F3 & Tissue factor & 10 \\
\hline P30837 & ALDH1B1 & Aldehyde dehydrogenase X, mitochondrial & 7 \\
\hline 000748 & CES2 & Cocaine esterase & 7 \\
\hline P05231 & IL-6 & Interleukin-6 & 7 \\
\hline P07237 & P4HB & Protein disulfide-isomerase & 7 \\
\hline
\end{tabular}

\subsection{Outcome of PPI network}

The PPI network was constructed from the common 78 targets (Fig. 4). Due to the lack of any interactions with other targets, Chymotrypsin-C (CTRC) and Alpha-ketoglutarate-dependent dioxygenase (FTO) were removed from the network. Therefore, the network contained 76 nodes and 376 edges with the average degree value of 9.64. The target proteins with higher degree value than average degree value were Interleukin-6 (IL-6), Vascular endothelial growth factor A (VEGFA), Estrogen receptor (ESR1), Hypoxia-inducible factor 1-alpha (HIF1A), Matrix metalloproteinase-9 (MMP9), Amyloid-beta precursor protein (APP), cAMP responsive element binding protein 1 (CREB1), Heat shock protein family A member 5 (HSPA5), Transcription factor p65 (RELA) and Interleukin-2 (IL-2). The 10 targets were indispensable for constructing interaction between protein and protein in the network, and were considered as the putative key target of SGXZ decoction for the treatment of NAFLD.

\subsection{GO enrichment analysis}

Gene ontology (G0) functionally annotated targets in PPI network into three main aspects containing molecular function (MF), cellular component (CC) and biological process (BP). In total, $185 \mathrm{GO}$ entries including 45 of MF , 25 of $\mathrm{CC}$ and 115 of BP were acquired based on the $P$ value (P凶0.05). For BP, the targets were mainly concentrated on the oxidation-reduction process, positive regulation of angiogenesis, negative regulation of apoptotic process, regulation of insulin-like factor receptor signaling pathway and 
so on. For CC, the targets were mainly responsible for the extracellular space, extracellular exosome, cell surface, extracellular region, mitochondrion, extracellular matrix and so on. In regard to MF, the intersection targets were distributed in enzyme binding, insulin-like growth factor binding, electron carrier activity, oxidoreductase activity, receptor binding, protein heterodimerization activity, iron ion binding, glycosphingolipid binding and protein binding. The bubble plot and histogram of the most significant enriched GO terms were exhibited in Figure 5.

\subsection{KEGG enrichment analysis}

The 43 annotated pathways were totally obtained based on the targets in PPI network, and the top 20 of them with smallest significance value were shown in Figure. $6 \mathrm{~A}$ and Table 3. The bubble plot of the pathways suggested a concentration on the insulin resistance, amino acid metabolism, cancer-related pathways, inflammation-related pathways including MAPK signaling pathway, HIF-1 signaling pathway, TNF signaling pathway and PI3K-Akt pathway. Moreover, the common targets also concentrated on the endocrine participated biological process such as estrone signaling pathway and immunological pathway of T cell receptor. To reveal the network interaction of the pathways and the involved targets, target-pathway network was constructed and analyzed which consisted of 58 nodes and 130 edges as shown in Figure 6B, indicating a complicated interaction among them. Pathways with largest degree including pathways in cancer, PI3K-Akt, MAPK signaling pathway, proteoglycan in cancer and HIF-1 pathway, suggesting their significant role in the treatment of SGXZ decoction on NAFLD.

Table 3

Annotation of KEGG pathways and the involved potential targets 


\begin{tabular}{|c|c|c|c|c|}
\hline KEGG ID & Term & Count & $P$ value & Targets \\
\hline hsa04931 & Insulin resistance & 8 & 7.75E-05 & $\begin{array}{l}\text { RPS6KA3, PTPN1, IL-6, CREB1, } \\
\text { PRKCE, PTPN11, RELA, NFKB1 }\end{array}$ \\
\hline hsa04066 & HIF-1 signaling pathway & 7 & 3.17E-04 & $\begin{array}{l}\text { IL-6, SERPINE1, PRKCA, HIF1A, RELA, } \\
\text { NFKB1, VEGFA }\end{array}$ \\
\hline hsa00380 & Tryptophan metabolism & 5 & 5.77E-04 & $\begin{array}{l}\text { MAOB, ALDH2, ALDH1B1, MAOA, } \\
\text { CYP1A1 }\end{array}$ \\
\hline hsa04726 & Serotonergic synapse & 7 & $6.91 \mathrm{E}-04$ & $\begin{array}{l}\text { APP, CYP2C8, MAOB, MAOA, ALOX5, } \\
\text { PRKCA, ALOX } 12\end{array}$ \\
\hline hsa00340 & Histidine metabolism & 4 & 0.001191 & MAOB, ALDH2, ALDH1B1, MAOA \\
\hline hsa05030 & Cocaine addiction & 5 & 0.001253 & CREB1, MAOB, MAOA, RELA, NFKB1 \\
\hline hsa05200 & Pathways in cancer & 12 & 0.001302 & $\begin{array}{l}\text { IL-6, MMP1, MMP2, RARA, PRKCA, } \\
\text { IKBKG, FGF1, HIF1A, MMP9, RELA, } \\
\text { NFKB1, VEGFA }\end{array}$ \\
\hline hsa00330 & $\begin{array}{l}\text { Arginine and proline } \\
\text { metabolism }\end{array}$ & 5 & 0.001352 & $\begin{array}{l}\text { MAOB, P4HA1, ALDH2, ALDH1B1, } \\
\text { MAOA }\end{array}$ \\
\hline hsa05134 & Legionellosis & 5 & 0.001803 & IL-6, RELA, NFKB1, HSPD1, HSPA1A \\
\hline hsa05161 & Hepatitis B & 7 & 0.002742 & $\begin{array}{l}\text { IL-6, CREB1, PRKCA, IKBKG, MMP9, } \\
\text { RELA, NFKB1 }\end{array}$ \\
\hline hsa04010 & $\begin{array}{l}\text { MAPK signaling } \\
\text { pathway }\end{array}$ & 9 & 0.002986 & $\begin{array}{l}\text { RPS6KA3, NR4A1, PRKCA, IKBKG, } \\
\text { MAPT, FGF1, RELA, NFKB1, HSPA1A }\end{array}$ \\
\hline hsa05205 & Proteoglycans in cancer & 8 & 0.003139 & $\begin{array}{l}\text { MMP2, PTPN6, PTPN11, PRKCA, } \\
\text { HIF1A, ESR1, MMP9, VEGFA }\end{array}$ \\
\hline hsa05142 & $\begin{array}{l}\text { Chagas disease } \\
\text { (American } \\
\text { trypanosomiasis) }\end{array}$ & 6 & 0.003296 & $\begin{array}{l}\text { IL-6, SERPINE1, IKBKG, RELA, NFKB1, } \\
\text { IL-2 }\end{array}$ \\
\hline
\end{tabular}

\begin{tabular}{|c|c|c|c|c|}
\hline hsa04668 & TNF signaling pathway & 6 & 0.003726 & $\begin{array}{l}\text { IL-6, CREB1, IKBKG, MMP9, RELA, } \\
\text { NFKB1 }\end{array}$ \\
\hline hsa05202 & $\begin{array}{l}\text { Transcriptional } \\
\text { misregulation in cancer }\end{array}$ & 7 & 0.005495 & $\begin{array}{l}\text { IL-6, BCL2A1, IGFBP3, RARA, MMP9, } \\
\text { RELA, NFKB1 }\end{array}$ \\
\hline hsa04151 & $\begin{array}{l}\text { PI3K-Akt signaling } \\
\text { pathway }\end{array}$ & 10 & 0.005885 & $\begin{array}{l}\text { NR4A1, IL-6, CREB1, PRKCA, IKBKG, } \\
\text { FGF1, RELA, NFKB1, IL-2, VEGFA }\end{array}$ \\
\hline hsa05219 & Bladder cancer & 4 & 0.007229 & MMP1, MMP2, MMP9, VEGFA \\
\hline hsa05215 & Prostate cancer & 5 & 0.010389 & $\begin{array}{l}\text { CREB1, SRD5A2, IKBKG, RELA, } \\
\text { NFKB1 }\end{array}$ \\
\hline hsa04915 & $\begin{array}{l}\text { Estrogen signaling } \\
\text { pathway }\end{array}$ & 5 & 0.015491 & $\begin{array}{l}\text { CREB1, MMP2, ESR1, MMP9, } \\
\text { HSPA1A }\end{array}$ \\
\hline hsa04660 & $\mathrm{T}$ cell receptor signaling & 5 & 0.016021 & PTPN6, IKBKG, RELA, NFKB1, IL-2 \\
\hline
\end{tabular}




\subsection{Verification of the binding between key compounds and targets}

Molecular docking was employed to verify the binding between the key compounds with key targets, revealing the potential therapeutic effect of SGXZ decoction acting on these targets. Crystal structures of the targets were downloaded from PDB database except SLC5A1, CES2 and ALDH1B1 for their lack of 3D structures. Homology modeling was used to build the structures of SLC5A1 and ALDH1B1 according to previous studies [50-51] (Supplementary Figure 1). However, the crystal structure of CREB1 could be obtained neither from the PDB database nor through the homology modeling since none of templates with good quality existed. Besides, 3 duplicated key targets between compound-target-disease network and PPI network were removed. Thus, a total of 10 key compounds were docked into 16 key targets. After docking, 160 pair of compound-target complexes were retained according to their best binding affinity, and the heat map of their docking energy was shown in Figure 7. Moreover, the binding energy of each pair of compound-target complex was provided in Supplementary Table 3. Binding energy with negative value indicated that the ligand molecule was able to combine with the receptor target proteins. The lower and more negative binding energy suggested a better binding affinity of active compounds with target proteins. Almost half of the key compound could combine with most of the targets. Among them, gallic acid (SX01), chlorogenic acid (SX07) and isochlorogenic acid A (SX13) could bind into all the key targets with the lowest binding energy. The accurate values of binding energy between these three key active compounds and key targets were shown in Table 4. Isochlorogenic acid A had the most negative binding energy with HSPA5 $(-53.91 \mathrm{kcal} / \mathrm{mol})$, followed by the RELA $(-51.73 \mathrm{kcal} / \mathrm{mol}), \mathrm{MMP9}(-48.88 \mathrm{kcal} / \mathrm{mol})$, SLC5A1 (-34.57 kcal/mol), HIF1A (-39.94 kcal/mol) and FGF1 (-38.21 kcal/mol). Chlorogenic acid also showed great binding affinity with HSPA5 $(-43.27 \mathrm{kcal} / \mathrm{mol})$. Gallic acid exhibited with the most negative binding energy with ESR1 (-35.57 kcal/mol), followed by HSPA5 (-33.25 kcal/mol) and RELA (-32.32 $\mathrm{kcal} / \mathrm{mol})$. Residues that produce hydrogen bonds shared by all 3 key active compounds with target were also listed in the Table 4. Moreover, the interaction between the best poses of three compounds and targets were displayed in Figure. 8. The results of molecular docking revealed that gallic acid, chlorogenic acid and isochlorogenic acid A might be the most potentially active ingredients of SGXZ decoction treating NAFLD.

Table 4

Binding energy between key active compounds and targets $(\mathrm{kcal} / \mathrm{mol})$ 


\begin{tabular}{|c|c|c|c|c|c|}
\hline \multirow{2}{*}{$\begin{array}{l}\text { Target } \\
\text { proteins }\end{array}$} & \multicolumn{3}{|c|}{ Ingredients } & \multirow{2}{*}{$\begin{array}{l}\text { Average binding } \\
\text { energy }\end{array}$} & \multirow{2}{*}{$\begin{array}{l}\text { Hydrogen Bond Interactions } \\
\text { (Amino acid) }\end{array}$} \\
\hline & SX01 & SX07 & sX13 & & \\
\hline FGF1 & -27.07 & -22.06 & -38.21 & -29.11 & $\begin{array}{l}\text { LYS112, LYS113, ASN18, LYS128, } \\
\text { LYS118 }\end{array}$ \\
\hline VEGFA & -21.22 & -18.94 & -29.06 & -23.07 & PR053, GLU30, \\
\hline IL-2 & -19.90 & -15.25 & -29.85 & -21.67 & LYS35 \\
\hline LGALS3 & -22.81 & -17.71 & -34.94 & -25.15 & ASN174, GLU184 \\
\hline SLC5A1 & -27.92 & -31.68 & -44.10 & -34.57 & SER77, ASP294, GLN295 \\
\hline F3 & -24.94 & -5.43 & -19.86 & -16.74 & LYS65, LYS46 \\
\hline ALDH1B1 & -29.62 & -28.87 & -24.23 & -27.57 & CYS302, CYS303 \\
\hline CES2 & -27.76 & -23.35 & -32.97 & -28.03 & SER228 \\
\hline IL-6 & -26.37 & -17.42 & -30.38 & -27.72 & $\mathrm{~N} / \mathrm{A}$ \\
\hline $\mathrm{P} 4 \mathrm{HB}$ & -19.07 & -12.82 & -33.19 & -21.69 & TRP396 \\
\hline ESR1 & -35.57 & -24.86 & -3.86 & -21.43 & GLU353, LEU346 \\
\hline MMP9 & -23.77 & -25.52 & -48.88 & -32.72 & HIS230, ALA191 \\
\hline HIF1A & -28.66 & -28.22 & -39.94 & -32.27 & THR196, HIS199 \\
\hline APP & -25.31 & -21.32 & -32.41 & -26.35 & LYS447, ASP444, HIS439 \\
\hline HSPA5 & -33.25 & -43.27 & -53.91 & -43.48 & $\begin{array}{l}\text { GLY364, SER365, LYS296, GLU293, } \\
\text { ARG297 }\end{array}$ \\
\hline RELA & -32.32 & -30.32 & -51.73 & -38.12 & ALA222, ALA73, TYR75 \\
\hline
\end{tabular}

\section{Discussion}

NAFLD is a metabolic related syndrome characterized as dysfunctional hepatic lipid metabolism and insulin resistance [52-53], which has been considered the fastest growing cause of HCC [54] and strongly associated with the increasing risks of type 2 diabetes, cardiovascular disease, chronic kidney disease and hypertension [55-57]. Currently, no pharmacological therapies were approved by FDA in the treatment of NAFLD and seeking an effective agent is urgently desiderated. TCM has been proved to treat NAFLD with the unique advantages of holistic concept and differentiation treatment, as well as mechanisms of multi-target and multi-channel action [27-58-59](34344394; 31558860; 33639194). Shugan Xiaozhi (SGXZ) decoction was proposed and designed with insights gained from two classic prescriptions of TCM - "Sini decoction" and "Yinchenhao decoction", and showed a good efficacy for the treatment of NAFLD [32-60]. In addition, both of Sini decoction and Yinchenhao decoction exerted 
therapeutic effect for NAFLD and NASH [61-63]. Nevertheless, the underlying therapeutic mechanisms of SGXZ decoction remained to be further elucidated.

In the present study, active compounds of SGXZ decoction with corresponding targets were firstly identified, and the overlapping targets between SGXZ decoction and NAFLD were considered to be the main targets these compounds acted on. Moreover, compounds including series of phenolic acids and flavonoids were selected as the key bioactive ingredients for the treatment of SGXZ decoction on NAFLD based on their contributions in the compound-target-disease network (Figure2, Table 1). Previous study indicated that the intake of phenolic acids alleviated hepatic steatosis, reduced fibrosis and the insulin resistance in NAFLD patients [64]. Gallic acid, a simple polyphenol, was reported to reduce lipid accumulation that is related to $\beta$-oxidation and ketogenesis [65]. Specifically, the hepatoprotective effect of gallic acid attributed to the repression of inflammatory signaling pathways including nuclear factor-kB (NF-KB)/TNF- $\alpha$ /IL-6 and ROS/NF-KB /TNF- $a$ in NAFLD rats [66-67]. Chlorogenic acid, a natural polyphenol extracted from Artemisiae Scopariae (Yin-Chen in Chinese) [68] and Lonicera japonica (JinYin-Hua) [69], could ameliorate HFD-induced hepatic steatosis and inflammation via inhibition of TNF-a and IL- 6 in liver, which was associated with regulation of gut microbiota and an increase of Glucagon-like peptide-1 secretion [70-71]. Isochlorogenic acid A was suggested to possess properties of hepatoprotective and anti-hepatitis B through suppressing oxidation [72]. Moreover, it was indicated that isochlorogenic acid $A$ exerted a protective effect on liver fibrosis through inhibiting inflammation via HMGB1/TLR4/NF-kB signaling pathways [73]. In addition, flavonoids are natural products widely distributed in plants, exhibiting not only antidiabetic and hypoglycemic activities but also antiinflammatory and antioxidant properties, and were recognized to have protective effect in the treatment of NAFLD [74-76]. As a kind of flavonoid, liquiritin could ameliorate cyclophosphamide-induced liver injury and inflammation by inhibiting the elevated MMP-9 expression, hepatic infiltration of neutrophils, myeloperoxidase activity, IL-6 mRNA expression and NF-KB activation [77]. Naringin is a flavanone glycoside isolated from Aurantii Fructus Immaturus (Zhi-Shi) [78] and has been proved to improve lipid metabolism disorders through reducing hepatic lipid accumulation in tissue-engineered NAFLD model [79]. Neohesperidin could induce the PGC-1a expression through activating AMP-activated protein kinase (AMPK), increasing hepatic mitochondrial biogenesis and fatty acid oxidation in NAFLD mice [80]. To sum up, three phenolic acids (gallic acid, chlorogenic acid, isochlorogenic acid A) and three flavonoids (liquiritin, naringin, neohesperidin) with hepatoprotective effect could serve as the potentially main active ingredients of SGXZ decoction for the treatment of NAFLD.

Together with key targets in compound-target-disease network (Figure 3, Table 2), 10 targets with highest degree in the PPI network were as well recognized as core targets of SGXZ decoction treating NAFLD (Figure 4). The target protein level of VEGFA, FGF1, IL-2, LGALS3, SLC5A1 and IL-6 were excessively expressed in compound-target-disease network. Moreover, IL-6 possessed the highest degree in PPI network, followed by VEGFA, ESR1, HIF1A, MMP9, HSPA5, RELA and IL-2. Particularly, IL-6, IL-2 and VEGFA contributed significantly to the construction of both compound-target-disease and PPI networks, indicating their critical role among the targets. IL- 6 has been considered to cause inflammatory properties and a vital factor associated with the pathology of metabolic disorders [81]. Numerous studies have 
highlighted that increased IL-6 level promoted hepatic insulin resistance [82] and impaired the lipid metabolism [83]. IL-6 deficiency ameliorated the hepatic inflammation and injury in NASH mice fed with methionine and choline-deficient diet [84]. Similarly, upregulated IL-2 potentially leads to the increased insulin resistance as well as several other metabolic inflammatory markers in obese population [85]. VEGFA is a major proangiogenic cytokine regulating angiogenesis. Increased VEGFA was reported in liver of animal model and serum of NASH patients [86-87], which accelerates angiogenesis and therefore drives hepatic inflammation and fibrosis in NAFLD [87].

Hepatic steatosis and lipid accumulation could produce a hypoxic proangiogenic microenvironment, while the respond to this condition was mediated by hypoxia-inducible factors (HIFs) [88-89]. Hypoxia upregulated the HIF1A expression in liver, leading to the aggravated steatosis by suppression of fatty acid (FFA) $\beta$-oxidation and by induction of FFA uptake and inflammatory factors [89-90]. Furthermore, HIF1A promoted liver fibrosis in NAFLD by activating PTEN/ NF-KB p65 signaling pathway [91]. RELA, also known as p65, is one of the five members in NF-KB family and is a pivotal transcription factor regulating inflammatory molecules [92]. Inhibition of NF-KB signaling alleviated hepatic lipid accumulation and hepatic inflammation in NAFLD [93-94]. Moreover, abnormal lipid deposition as well as insulin resistance in NAFLD often leads to endoplasmic reticulum (ER) stress, which further triggers the unfolded protein response and thereby causes inflammation in hepatocytes [95]. GRP78 (HSPA5) is a chaperone heat shock protein playing the central role in maintaining ER proteostasis under excessive stress [96]. Decreased expression of ER stress molecule GRP78 contributed to reducing fasting glucose and lipid profile, and attenuating NAFLD [97-99]. LGALS3 has been shown to participate in glucose intolerance and lipid metabolism disorders, which is an essential regulator of insulin resistance, fibrosis and inflammation cytokines including TNF- $\alpha$, IL-6 and IL-1 $\beta$ [100-101]. FGF1 exerts a protective role in series of metabolic disorders [102]. Investigations revealed that FGF1 could reduce blood glucose and ameliorate hepatic steatosis, inflammation and fibrosis through modulation of oxidative stress and ER stress [103-104]. The onset of hepatic inflammation caused the fibrogenesis in NAFLD, which was manifested by deposited extracellular matrix (ECM) proteins including collagens, elastin and fibronectin [105-106]. MMP-9 performs the vital role in modulating and degrading gelatins, collagens and other ECM compounds [107-108]. A decreased MMP-9 was associated with more advanced fibrosis and serum liver injury indices (AST, GGT) in NAFLD patients, while increased MMP-9 activity could precede the clearance of fibrotic matrix [109-110]. SLC5A1 encodes the sodium glucose cotransporter 1 (SGLT1), and inhibition of SGLT1 not only contributed to regulate hepatic glucose metabolism but also mitigate development and progression of NAFLD [111-112]. To conclude, it was assumed that SGXZ decoction might perform comprehensive regulations of anti-inflammation, anti-lipid deposition, anti-insulin resistance, anti-fibrosis, and anti-ER stress on NAFLD through these key targets.

After that, GO functional enrichment analysis revealed that the targets in PPI network mainly involved oxidation, positive regulation of angiogenesis, metabolic process, hypoxia, extracellular matrix and insulin-like factor binding, and other biological processes (Figure 5). The results of GO analysis coincided with the indispensable contribution of aforementioned key targets among all targets in PPI network. In addition, KEGG enrichment analyses indicated that the action pathway mainly included insulin resistance, 
amino acid metabolism, cancer-related pathways, inflammation-related pathways, estrone signaling pathway and T cell receptor pathway (Figure 6A, Table 3). As shown in Figure. 6B, the 'target-pathway' interaction network suggested that bioactive compounds in SGXZ decoction performed therapeutic role in regulation of NAFLD through multi-targets and multi-pathways. Through literature retrieval, six signaling pathways including PI3K-Akt, MAPK, insulin resistance, HIF-1, Tryptophan metabolism and Estrogen signaling pathway were recognized as core pathways involved in the treatment of SGXZ decoction on NAFLD. The six core pathways and their interaction were cited from KEGG database and displayed in Figure 9. Among the core pathways, PI3K-Akt, MAPK and HIF-1 signaling pathway were associated with inflammation that is the driving force for the development and evolution of NAFLD [113-114]. Researches provided evidence that regulation of PI3K-Akt signaling mediators could improve hepatocyte damage, hepatic gluconeogenesis and lipid disorder [115-117]. Moreover, inhibiting apoptosis and promoting autophagy via the reactive oxygen species (ROS)/MAPK pathway significantly decreased total cholesterol and triglyceride levels of both plasma and liver in high fat diet-fed mice [118]. In addition, increased ROS formation, triglyceride and lipid accumulation in hepatocyte leaded to excessive oxidative stress and inflammatory responses that further produced a hypoxic microenvironment [88-119] (33272734, 33804956). HIF-1 signaling acts as the key response to hypoxia. Loss of HIF-1a (HIF1A) activates the oxidative stress, promoted the lipid deposition and the secretion of pro-inflammatory IL- 6 and TNF-a, suggesting its regulatory role in the progression of NAFLD [119-120](33272734, 30242180). Indeed, increased FFAs and lipogenesis in hepatocytes, release of proinflammatory cytokines from adipose tissue such as IL- 6 and TNF- $a$, together with altered gut microbiota gave rise to the insulin resistance, which predisposes to the development of NAFLD and progression to NASH [121-123]. Tryptophan is a kind of amino acid that could produce indoles by bacterial enzyme trytophanase $A$ in intestinal epithelial cell. Indoles promote intestinal barrier function and could translocate from the intestinal to the liver to modulate hepatic lipid metabolism and inflammation to protect against NAFLD [124]. Moreover, the hepatic metabolism and inflammation in the onset and progression of NAFLD showed a sex difference, which was potentially due to different estrogen signaling activity [125-126]. Estrogen deficiency increased risk for liver fibrosis, and postmenopausal women were more likely to develop NAFLD than men [127-128]. In general, the above six core pathways and their interaction were correlated to NAFLD and might be acted on by SGXZ decoction in the treatment of NAFLD.

In the end, molecular docking (Table 4, Figure 8) showed that most of the key compounds could be docked into the key protein targets. Moreover, bioactive compounds of isochlorogenic acid A, chlorogenic acid and gallic acid could bind best with all of the key targets, especially with HSPA5, RELA, MMP9, SLC5A1, HIF1A and FGF1. The results of molecular docking simulations provided a putative pharmacological activity for the treatment of SGXZ decoction on NAFLD. Our present study applied network pharmacology combined with molecular docking to predict the key active ingredients and pathways of SGXZ decoction in NAFLD treatment, which still need to be verified by future experimental validations. 


\section{Conclusions}

In this study, network pharmacology approach combined with molecular docking method were performed to elucidate the underlying mechanisms, including the putative active ingredients, key targets and pivotal signaling pathways, of SGXZ decoction in the treatment of NAFLD. Among the predicted key compounds, three bioactive ingredients of SGXZ, isochlorogenic acid A, chlorogenic acid and gallic acid might exert the essential pharmacological effect. Furthermore, VEGFA, IL-6, IL-2, HSPA5, RELA, MMP9, SLC5A1, HIF1A and FGF1 were assumed to be the key targets acted on by those bioactive ingredients. In addition, signaling pathways of inflammation, oxidative stress, insulin resistance, intestinal barrier function, and lipid metabolism were mainly involved. The results provided an insight into the therapeutic strategies of NAFLD and evidence for future research. Nonetheless, more experimental approaches should be carried out to validate the findings.

\section{Abbreviations}

TCM: Traditional Chinese medicine; NAFLD: Nonalcoholic fatty liver disease; SGXZ: Shugan Xiaozhi decoction; SMILES: Simplified molecular input line entry system; SEA: Similarity ensemble approach; PDB: RCSB PROTEIN DATA BANK; PPI: Protein-protein interaction; STRING: Search Tool for the Retrieval of Interacting Genes/Proteins; DS: Discovery studio software 2016; GO: Gene ontology; BP: Biological process; CC: Cell components; MF: Molecular functions; KEGG: Kyoto Encyclopedia of Genes and Genomes; FFA: Free fatty acid

\section{Declarations}

\section{Acknowledgements}

Not applicable.

\section{Authors' contributions}

YR and XYF conceived and designed the study. YR collected and acquired the data. YHL, JDS, XLY and FL performed the data analysis, RY wrote the first version of manuscript, YHL made the critical revision, and $\mathrm{XYF}$ finalized the manuscript. All authors are responsible for reviewing and evaluating the data. All authors read and approved the final manuscript.

\section{Funding}

This work is financially supported by the Shenzhen Natural Science Foundation (grant No. JCYJ20190812161605538) and Sanming Project of Medicine in Shenzhen (grant No. SZSM201612074). 


\section{Availability of supporting data}

All data are available in the manuscript and they are showed in figures, tables and supplementary files.

\section{Ethical approval and consent to participate}

Not applicable.

\section{Consent for publication}

Not applicable.

\section{Competing interests}

The authors declare no conflicts of interest in relation to this work.

\section{References}

1. Challa TD, Wueest $S$, Lucchini FC, et al. Liver ASK1 protects from non-alcoholic fatty liver disease and fibrosis. EMBO Mol Med. 2019;11:e10124.

2. Raza S, Rajak S, Upadhyay A, et al. Current treatment paradigms and emerging therapies for NAFLD/NASH. Front Biosci (Landmark Ed). (2021); 26: 206-237.

3. Tilg $\mathrm{H}$, Effenberger M. From NAFLD to MAFLD: when pathophysiology succeeds. Nat Rev Gastroenterol Hepatol. 2020;17:387-8.

4. Liu Z, Zhang Y, Graham S, et al. Causal relationships between NAFLD, T2D and obesity have implications for disease subphenotyping. J Hepatol. 2020;73:263-76.

5. Stefan N, Häring HU, Cusi K. Non-alcoholic fatty liver disease: causes, diagnosis, cardiometabolic consequences, and treatment strategies. Lancet Diabetes Endocrinol. 2019;7:313-24.

6. Mundi MS, Velapati S, Patel J, et al. Evolution of NAFLD and Its Management. Nutr Clin Pract. 2020;35:72-84.

7. Zhou J, Zhou F, Wang W, et al. Epidemiological Features of NAFLD From 1999 to 2018 in China. Hepatology. 2020;71:1851-64.

8. Younossi ZM, Blissett D, Blissett R, et al. The economic and clinical burden of nonalcoholic fatty liver disease in the United States and Europe. Hepatology. 2016;64:1577-86.

9. Younossi Z, Stepanova M, Ong JP, et al. Nonalcoholic Steatohepatitis Is the Fastest Growing Cause of Hepatocellular Carcinoma in Liver Transplant Candidates. Clin Gastroenterol Hepatol. 2019;17:748-55.e743. 
10. Jeznach-Steinhagen A, Ostrowska J, Czerwonogrodzka-Senczyna A, et al. Dietary and Pharmacological Treatment of Nonalcoholic Fatty Liver Disease. Medicina (Kaunas). (2019); 55.

11. Centis E, Moscatiello S, Bugianesi E, et al. Stage of change and motivation to healthier lifestyle in non-alcoholic fatty liver disease. J Hepatol. 2013;58:771-7.

12. Kantartzis K, Thamer C, Peter A, et al. High cardiorespiratory fitness is an independent predictor of the reduction in liver fat during a lifestyle intervention in non-alcoholic fatty liver disease. Gut. 2009;58:1281-8.

13. Eight-year weight. losses with an intensive lifestyle intervention: the look AHEAD study. Obesity (Silver Spring). (2014); 22: 5-13.

14. Konerman MA, Jones JC, Harrison SA. Pharmacotherapy for NASH: Current and emerging. J Hepatol. 2018;68:362-75.

15. Takahashi Y, Sugimoto K, Inui H, et al. Current pharmacological therapies for nonalcoholic fatty liver disease/nonalcoholic steatohepatitis. World J Gastroenterol. 2015;21:3777-85.

16. Ahsan F, Oliveri F, Goud HK, et al. Pleiotropic Effects of Statins in the Light of Non-Alcoholic Fatty Liver Disease and Non-Alcoholic Steatohepatitis. Cureus. 2020;12:e10446.

17. Nissen SE, Wolski K. Effect of rosiglitazone on the risk of myocardial infarction and death from cardiovascular causes. N Engl J Med. 2007;356:2457-71.

18. Miller ER 3rd, Pastor-Barriuso R, Dalal D, et al. Meta-analysis: high-dosage vitamin E supplementation may increase all-cause mortality. Ann Intern Med. 2005;142:37-46.

19. Klein EA, Thompson IM Jr, Tangen CM, et al. Vitamin E and the risk of prostate cancer: the Selenium and Vitamin E Cancer Prevention Trial (SELECT). Jama. 2011;306:1549-56.

20. Lincoff AM, Wolski K, Nicholls SJ, et al. Pioglitazone and risk of cardiovascular events in patients with type 2 diabetes mellitus: a meta-analysis of randomized trials. Jama. 2007;298:1180-8.

21. Liao HW, Saver JL, Wu YL, et al. Pioglitazone and cardiovascular outcomes in patients with insulin resistance, pre-diabetes and type 2 diabetes: a systematic review and meta-analysis. BMJ Open. 2017;7:e013927.

22. Pal S, Ghosh M, Ghosh S, et al. Atorvastatin induced hepatic oxidative stress and apoptotic damage via MAPKs, mitochondria, calpain and caspase12 dependent pathways. Food Chem Toxicol. 2015;83:36-47.

23. Abdoli N, Azarmi Y, Eghbal MA. Protective Effects of N-acetylcysteine Against the Statins Cytotoxicity in Freshly Isolated Rat Hepatocytes. Adv Pharm Bull. 2014;4:249-54.

24. Fu K, Wang C, Ma C, et al. The Potential Application of Chinese Medicine in Liver Diseases: A New Opportunity. Front Pharmacol. 2021;12:771459.

25. Li C, Zhang H, Li X. The Mechanism of Traditional Chinese Medicine for the Treatment of Obesity. Diabetes Metab Syndr Obes. 2020;13:3371-81.

26. Zhang HY, Tian JX, Lian FM, et al. Therapeutic mechanisms of traditional Chinese medicine to improve metabolic diseases via the gut microbiota. Biomed Pharmacother. 2021;133:110857. 
27. Dai X, Feng J, Chen Y, et al. Traditional Chinese Medicine in nonalcoholic fatty liver disease: molecular insights and therapeutic perspectives. Chin Med. 2021;16:68.

28. Liang Y, Lin C, Huang S, et al. Traditional Chinese Medicine and Intestinal Microbiota: A Complementary and Integrative Health Approach to Ameliorate Obesity-Related Diseases. Holist Nurs Pract. 2019;33:259-65.

29. Ma J, Zhou Q, Li H. Gut Microbiota and Nonalcoholic Fatty Liver Disease: Insights on Mechanisms and Therapy. Nutrients. (2017); 9.

30. Tang HH, Wei CS, Zheng YJ, et al. Evaluating the Clinical Effect of Modified Shugan Xiaozhi Decoction in Treating Nonalcoholic Fatty Liver Disease Based on Fibroscan Technique. Journal of Traditional Chinese Medicine. 2018;59:594-8.

31. Zhai FF, Sun H, Su XX, et al. Clinical efficacy of Shugan Xiaozhi granules in treatment of nonalcoholic steatohepatitis. Chinese Journal of Integrated Traditional Western Medicine on Liver Diseases. 2014;24:78-9+82.

32. Xing YF, Zhang Z, Fu WJ, et al Shugan Xiaozhi Decoction Attenuates Nonalcoholic Steatohepatitis by Enhancing PPARa and L-FABP Expressions in High-Fat-Fed Rats. Evid Based Complement Alternat Med. (2016); 2016: 7870189.

33. Zhai FF, Wang YQ, Feng L, et al. Study on the enterogenic mechanism of Shugan Xiaozhi decoction in treating NAFLD by regulating apoB48. Chinese Journal of Integrated Traditional and Western Medicine on Liver Diseases. (2021); 31: 721-725.

34. Liu ZH, Sun XB. Network pharmacology: new opportunity for the modernization of traditional Chinese medicine. Yao Xue Xue Bao. 2012;47:696-703.

35. Lai X, Wang X, Hu Y, et al. Editorial: Network Pharmacology and Traditional Medicine. Front Pharmacol. 2020;11:1194.

36. Zhang R, Zhu X, Bai H, et al. Network Pharmacology Databases for Traditional Chinese Medicine: Review and Assessment. Front Pharmacol. 2019;10:123.

37. Guo W, Huang J, Wang N, et al. Integrating Network Pharmacology and Pharmacological Evaluation for Deciphering the Action Mechanism of Herbal Formula Zuojin Pill in Suppressing Hepatocellular Carcinoma. Front Pharmacol. 2019;10:1185.

38. Zhang M, Yuan Y, Zhou W, et al. Network pharmacology analysis of Chaihu Lizhong Tang treating non-alcoholic fatty liver disease. Comput Biol Chem. 2020;86:107248.

39. Wang $X$, Wang ZY, Zheng JH, et al. TCM network pharmacology: A new trend towards combining computational, experimental and clinical approaches. Chin J Nat Med. 2021;19:1-11.

40. Zhou Z, Chen B, Chen S, et al Applications of Network Pharmacology in Traditional Chinese Medicine Research. Evid Based Complement Alternat Med. (2020); 2020: 1646905.

41. Jiao X, Jin X, Ma Y, et al. A comprehensive application: Molecular docking and network pharmacology for the prediction of bioactive constituents and elucidation of mechanisms of action in component-based Chinese medicine. Comput Biol Chem. 2021;90:107402. 
42. Álvarez-Carretero S, Pavlopoulou N, Adams J, et al. VSpipe, an Integrated Resource for Virtual Screening and Hit Selection: Applications to Protein Tyrosine Phospahatase Inhibition. Molecules. (2018); 23.

43. Keiser MJ, Roth BL, Armbruster BN, et al. Relating protein pharmacology by ligand chemistry. Nat Biotechnol. 2007;25:197-206.

44. Piñero J, Ramírez-Anguita JM, Saüch-Pitarch J, et al. The DisGeNET knowledge platform for disease genomics: 2019 update. Nucleic Acids Res. 2020;48:D845-d855.

45. Stelzer G, Rosen N, Plaschkes I, et al The GeneCards Suite: From Gene Data Mining to Disease Genome Sequence Analyses. Curr Protoc Bioinformatics. (2016); 54: 1.30.31-31.30.33.

46. Szklarczyk D, Gable AL, Lyon D, et al. STRING v11: protein-protein association networks with increased coverage, supporting functional discovery in genome-wide experimental datasets. Nucleic Acids Res. 2019;47:D607-d613.

47. Huang da W, Sherman BT, Lempicki RA. Systematic and integrative analysis of large gene lists using DAVID bioinformatics resources. Nat Protoc. 2009;4:44-57.

48. Huang da W, Sherman BT, Lempicki RA. Bioinformatics enrichment tools: paths toward the comprehensive functional analysis of large gene lists. Nucleic Acids Res. 2009;37:1-13.

49. Waterhouse A, Bertoni M, Bienert S, et al SWISS-MODEL: homology modelling of protein structures and complexes. Nucleic Acids Res. (2018); 46: W296-w303.

50. Wang L, Liu M, Yin F, et al. Trilobatin, a Novel SGLT1/2 Inhibitor, Selectively Induces the Proliferation of Human Hepatoblastoma Cells. Molecules. (2019); 24.

51. Song YQ, He RJ, Pu D, et al. Discovery and Characterization of the Biflavones From Ginkgo biloba as Highly Specific and Potent Inhibitors Against Human Carboxylesterase 2. Front Pharmacol. 2021;12:655659.

52. Kuchay MS, Choudhary NS, Mishra SK. Pathophysiological mechanisms underlying MAFLD. Diabetes Metab Syndr. 2020;14:1875-87.

53. Watt MJ, Miotto PM, De Nardo W, et al. The Liver as an Endocrine Organ-Linking NAFLD and Insulin Resistance. Endocr Rev. 2019;40:1367-93.

54. Burra P, Becchetti C, Germani G. NAFLD and liver transplantation: Disease burden, current management and future challenges. JHEP Rep. 2020;2:100192.

55. Targher G, Tilg H, Byrne CD. Non-alcoholic fatty liver disease: a multisystem disease requiring a multidisciplinary and holistic approach. Lancet Gastroenterol Hepatol. 2021;6:578-88.

56. Zhao YC, Zhao GJ, Chen Z, et al Nonalcoholic Fatty Liver Disease: An Emerging Driver of Hypertension. Hypertension. (2020); 75: 275-284.

57. Targher G, Byrne CD, Tilg H. NAFLD and increased risk of cardiovascular disease: clinical associations, pathophysiological mechanisms and pharmacological implications. Gut. 2020;69:1691-705. 
58. Yang JM, Sun Y, Wang M, et al. Regulatory effect of a Chinese herbal medicine formula on nonalcoholic fatty liver disease. World J Gastroenterol. 2019;25:5105-19.

59. Zhou $\mathrm{H}, \mathrm{Ma} \mathrm{C}$, Wang $\mathrm{C}$, et al. Research progress in use of traditional Chinese medicine monomer for treatment of non-alcoholic fatty liver disease. Eur J Pharmacol. 2021;898:173976.

60. Xing Y, F. and Tong GD. Experiences of Profeesor TONG Guang-dong in Treating Nonalcoholic Liver Disease. 14: JOURNAL OF LIAONING UNIVERSITY OF TCM; 2012. pp. 178-9.

61. Zhu F, Li YM, Feng TT, et al. Freeze-dried Si-Ni-San powder can ameliorate high fat diet-induced nonalcoholic fatty liver disease. World J Gastroenterol. 2019;25:3056-68.

62. Cheng F, Ma C, Wang X, et al. Effect of traditional Chinese medicine formula Sinisan on chronic restraint stress-induced nonalcoholic fatty liver disease: a rat study. BMC Complement Altern Med. 2017;17:203.

63. Chen SD, Fan Y, Xu WJ. Effects of yinchenhao decoction (see text) for non-alcoholic steatohepatitis in rats and study of the mechanism. J Tradit Chin Med. 2011;31:220-3.

64. Salomone F, Ivancovsky-Wajcman D, Fliss-Isakov N, et al. Higher phenolic acid intake independently associates with lower prevalence of insulin resistance and non-alcoholic fatty liver disease. JHEP Rep. 2020;2:100069.

65. Chao J, Cheng HY, Chang ML, et al. Gallic Acid Ameliorated Impaired Lipid Homeostasis in a Mouse Model of High-Fat Diet-and Streptozotocin-Induced NAFLD and Diabetes through Improvement of $\beta$ oxidation and Ketogenesis. Front Pharmacol. 2020;11:606759.

66. Fanaei H, Mard SA, Sarkaki A, et al. Gallic acid treats dust-induced NAFLD in rats by improving the liver's anti-oxidant capacity and inhibiting ROS/NFK $\beta / T N F a$ inflammatory pathway. Iran J Basic Med Sci. 2021;24:240-7.

67. Fanaei H, Mard SA, Sarkaki A, et al. Gallic acid protects the liver against NAFLD induced by dust exposure and high-fat diet through inhibiting oxidative stress and repressing the inflammatory signaling pathways NF-kß/TNF- $a /$ IL-6 in Wistar rats. Avicenna J Phytomed. 2021;11:527-40.

68. Cai Y, Zheng Q, Sun R, et al. Recent progress in the study of Artemisiae Scopariae Herba (Yin Chen), a promising medicinal herb for liver diseases. Biomed Pharmacother. 2020;130:110513.

69. Liu T, Yang J, Liu S, et al. Regulation of chlorogenic acid, flavonoid, and iridoid biosynthesis by histone H3K4 and H3K9 methylation in Lonicera japonica. Mol Biol Rep. 2020;47:9301-11.

70. Shi A, Li T, Zheng Y, et al. Chlorogenic Acid Improves NAFLD by Regulating gut Microbiota and GLP-1. Front Pharmacol. 2021;12:693048.

71. Zamani-Garmsiri F, Ghasempour G, Aliabadi M, et al. Combination of metformin and chlorogenic acid attenuates hepatic steatosis and inflammation in high-fat diet fed mice. IUBMB Life. 2021;73:25263.

72. Hao BJ, Wu YH, Wang JG, et al. Hepatoprotective and antiviral properties of isochlorogenic acid A from Laggera alata against hepatitis B virus infection. J Ethnopharmacol. 2012;144:190-4. 
73. Liu X, Huang K, Zhang RJ, et al. Isochlorogenic Acid A Attenuates the Progression of Liver Fibrosis Through Regulating HMGB1/TLR4/NF-kB Signaling Pathway. Front Pharmacol. 2020;11:582.

74. Bai L, Li X, He L, et al. Antidiabetic Potential of Flavonoids from Traditional Chinese Medicine: A Review. Am J Chin Med. 2019;47:933-57.

75. Yao H, Qiao YJ, Zhao YL, et al. Herbal medicines and nonalcoholic fatty liver disease. World J Gastroenterol. 2016;22:6890-905.

76. Wang K, Tan W, Liu X, et al. New insight and potential therapy for NAFLD: CYP2E1 and flavonoids. Biomed Pharmacother. 2021;137:111326.

77. Chen M, Zhang $\mathrm{C}$, Zhang J, et al. The involvement of DAMPs-mediated inflammation in cyclophosphamide-induced liver injury and the protection of liquiritigenin and liquiritin. Eur $\mathrm{J}$ Pharmacol. 2019;856:172421.

78. Wu J, Huang G, Li Y, et al. Flavonoids from Aurantii Fructus Immaturus and Aurantii Fructus: promising phytomedicines for the treatment of liver diseases. Chin Med. 2020;15:89.

79. Zhang X, Zhang Y, Gao W, et al. Naringin improves lipid metabolism in a tissue-engineered liver model of NAFLD and the underlying mechanisms. Life Sci. 2021;277:119487.

80. Wang SW, Sheng H, Bai YF, et al. Neohesperidin enhances PGC-1a-mediated mitochondrial biogenesis and alleviates hepatic steatosis in high fat diet fed mice. Nutr Diabetes. 2020;10:27.

81. Giraldez MD, Carneros D, Garbers C, et al. New insights into IL-6 family cytokines in metabolism, hepatology and gastroenterology. Nat Rev Gastroenterol Hepatol. 2021;18:787-803.

82. Akbari M, Hassan-Zadeh V. IL-6 signalling pathways and the development of type 2 diabetes. Inflammopharmacology. 2018;26:685-98.

83. Singh MK, Jayarajan R, Varshney S, et al. Chronic systemic exposure to IL 6 leads to deregulation of glycolysis and fat accumulation in the zebrafish liver. Biochim Biophys Acta Mol Cell Biol Lipids. 2021;1866:158905.

84. Mas E, Danjoux M, Garcia V, et al. IL-6 deficiency attenuates murine diet-induced non-alcoholic steatohepatitis. PLoS One. 2009;4:e7929.

85. Kochumon S, Al Madhoun A, Al-Rashed F, et al. Elevated adipose tissue associated IL-2 expression in obesity correlates with metabolic inflammation and insulin resistance. Sci Rep. 2020;10:16364.

86. Surapaneni KM, Vishnu Priya V, Mallika J. Effect of pioglitazone, quercetin, and hydroxy citric acid on vascular endothelial growth factor messenger RNA (VEGF mRNA) expression in experimentally induced nonalcoholic steatohepatitis (NASH). Turk J Med Sci. 2015;45:542-6.

87. Lefere S, Devisscher L, Geerts A. Angiogenesis in the progression of non-alcoholic fatty liver disease. Acta Gastroenterol Belg. 2020;83:301-7.

88. Lei L, Ei Mourabit $\mathrm{H}$, Housset $\mathrm{C}$, et al. Role of Angiogenesis in the Pathogenesis of NAFLD. J Clin Med. (2021); 10.

89. Isaza SC, Del Pozo-Maroto E, Domínguez-Alcón L, et al. Hypoxia and Non-alcoholic Fatty Liver Disease. Front Med (Lausanne). 2020;7:578001. 
90. Wu W, Li W, Wei J, et al. Chronic intermittent hypoxia accelerates liver fibrosis in rats with combined hypoxia and nonalcoholic steatohepatitis via angiogenesis rather than endoplasmic reticulum stress. Acta Biochim Biophys Sin (Shanghai). 2019;51:159-67.

91. Han J, He Y, Zhao H, et al. Hypoxia inducible factor-1 promotes liver fibrosis in nonalcoholic fatty liver disease by activating PTEN/p65 signaling pathway. J Cell Biochem. 2019;120:14735-44.

92. Gasparini C, Feldmann M. NF-kB as a target for modulating inflammatory responses. Curr Pharm Des. 2012;18:5735-45.

93. Daniel PV, Dogra S, Rawat P, et al. NF-kB p65 regulates hepatic lipogenesis by promoting nuclear entry of ChREBP in response to a high carbohydrate diet. J Biol Chem. 2021;296:100714.

94. Yang XD, Chen Z, Ye L, et al. Esculin protects against methionine choline-deficient diet-induced nonalcoholic steatohepatitis by regulating the Sirt1/NF-KB p65 pathway. Pharm Biol. 2021;59:922-32.

95. Lebeaupin C, Vallée D, Hazari Y, et al. Endoplasmic reticulum stress signalling and the pathogenesis of non-alcoholic fatty liver disease. J Hepatol. 2018;69:927-47.

96. Pobre KFR, Poet GJ, Hendershot LM. The endoplasmic reticulum (ER) chaperone BiP is a master regulator of ER functions: Getting by with a little help from ERdj friends. J Biol Chem. 2019;294:2098-108.

97. Sahin E, Bagci R, Bektur Aykanat NE, et al. Silymarin attenuated nonalcoholic fatty liver disease through the regulation of endoplasmic reticulum stress proteins GRP78 and XBP-1 in mice. J Food Biochem. 2020;44:e13194.

98. Nasiri-Ansari N, Nikolopoulou C, Papoutsi K, et al. Empagliflozin Attenuates Non-Alcoholic Fatty Liver Disease (NAFLD) in High Fat Diet Fed ApoE((-/-)) Mice by Activating Autophagy and Reducing ER Stress and Apoptosis. Int J Mol Sci. (2021); 22.

99. Bagci R, Sahinturk V, Sahin E. Azoramide ameliorates fructose-induced nonalcoholic fatty liver disease in mice. Tissue Cell. 2019;59:62-9.

100. Li YS, Li XT, Yu LG, et al. Roles of galectin-3 in metabolic disorders and tumor cell metabolism. Int $J$ Biol Macromol. 2020;142:463-73.

101. Yu H, Yang F, Zhong W, et al. Secretory Galectin-3 promotes hepatic steatosis via regulation of the PPARy/CD36 signaling pathway. Cell Signal. 2021;84:110043.

102. Nies VJ, Sancar G, Liu W, et al. Fibroblast Growth Factor Signaling in Metabolic Regulation. Front Endocrinol (Lausanne). 2015;6:193.

103. Xu Z, Wu Y, Wang F, et al. Fibroblast Growth Factor 1 Ameliorates Diabetes-Induced Liver Injury by Reducing Cellular Stress and Restoring Autophagy. Front Pharmacol. 2020;11:52.

104. Liu W, Struik D, Nies VJ, et al. Effective treatment of steatosis and steatohepatitis by fibroblast growth factor 1 in mouse models of nonalcoholic fatty liver disease. Proc Natl Acad Sci U S A. 2016;113:2288-93.

105. Munsterman ID, Kendall TJ, Khelil N, et al. Extracellular matrix components indicate remodelling activity in different fibrosis stages of human non-alcoholic fatty liver disease. Histopathology. 
2018;73:612-21.

106. Mas VR, Fisher RA, Archer KJ, et al. Proteomics and liver fibrosis: identifying markers of fibrogenesis. Expert Rev Proteomics. 2009;6:421-31.

107. Lachowski D, Cortes E, Rice A, et al. Matrix stiffness modulates the activity of MMP-9 and TIMP-1 in hepatic stellate cells to perpetuate fibrosis. Sci Rep. 2019;9:7299.

108. D'Amico F, Consolo M, Amoroso A, et al. Liver immunolocalization and plasma levels of MMP-9 in non-alcoholic steatohepatitis (NASH) and hepatitis C infection. Acta Histochem. 2010;112:474-81.

109. Goyale A, Jain A, Smith C, et al. Assessment of non-alcoholic fatty liver disease (NAFLD) severity with novel serum-based markers: A pilot study. PLoS One. 2021;16:e0260313.

110. Trojanek JB, Michałkiewicz J, Grzywa-Czuba R, et al Expression of Matrix Metalloproteinases and Their Tissue Inhibitors in Peripheral Blood Leukocytes and Plasma of Children with Nonalcoholic Fatty Liver Disease. Mediators Inflamm. (2020); 2020: 8327945.

111. David-Silva A, Esteves JV, Morais M, et al. Dual SGLT1/SGLT2 Inhibitor Phlorizin Ameliorates NonAlcoholic Fatty Liver Disease and Hepatic Glucose Production in Type 2 Diabetic Mice. Diabetes Metab Syndr Obes. 2020;13:739-51.

112. Honda Y, Ozaki A, Iwaki M, et al. Protective effect of SGL5213, a potent intestinal sodium-glucose cotransporter 1 inhibitor, in nonalcoholic fatty liver disease in mice. J Pharmacol Sci. 2021;147:17683.

113. Byun KA, Oh S, Son M, et al. Dieckol Decreases Caloric Intake and Attenuates Nonalcoholic Fatty Liver Disease and Hepatic Lymphatic Vessel Dysfunction in High-Fat-Diet-Fed Mice. Mar Drugs. (2021); 19.

114. Sutti S, Albano E. Adaptive immunity: an emerging player in the progression of NAFLD. Nat Rev Gastroenterol Hepatol. 2020;17:81-92.

115. Yang $P$, Liang $Y$, Luo $Y$, et al. Liraglutide ameliorates nonalcoholic fatty liver disease in diabetic mice via the IRS2/PI3K/Akt signaling pathway. Diabetes Metab Syndr Obes. 2019;12:1013-21.

116. Chen SH, Liu XN, Peng Y. MicroRNA-351 eases insulin resistance and liver gluconeogenesis via the PI3K/AKT pathway by inhibiting FLOT2 in mice of gestational diabetes mellitus. J Cell Mol Med. 2019;23:5895-906.

117. Liao $X$, Song $L$, Zhang $L$, et al. LAMP3 regulates hepatic lipid metabolism through activating PI3K/Akt pathway. Mol Cell Endocrinol. 2018;470:160-7.

118. Wu D, Liu Z, Wang Y, et al Epigallocatechin-3-Gallate Alleviates High-Fat Diet-Induced Nonalcoholic Fatty Liver Disease via Inhibition of Apoptosis and Promotion of Autophagy through the ROS/MAPK Signaling Pathway. Oxid Med Cell Longev. (2021); 2021: 5599997.

119. He Y, Yang W, Gan L, et al. Silencing HIF-1a aggravates non-alcoholic fatty liver disease in vitro through inhibiting PPAR-a/ANGPTL4 singling pathway. Gastroenterol Hepatol. 2021;44:355-65.

120. Arai T, Tanaka M, Goda N. HIF-1-dependent lipin1 induction prevents excessive lipid accumulation in choline-deficient diet-induced fatty liver. Sci Rep. 2018;8:14230. 
121. Buzzetti E, Pinzani M, Tsochatzis EA. The multiple-hit pathogenesis of non-alcoholic fatty liver disease (NAFLD). Metabolism. 2016;65:1038-48.

122. Khan RS, Bril F, Cusi K, et al. Modulation of Insulin Resistance in Nonalcoholic Fatty Liver Disease. Hepatology. 2019;70:711-24.

123. Lim JS, Mietus-Snyder M, Valente A, et al. The role of fructose in the pathogenesis of NAFLD and the metabolic syndrome. Nat Rev Gastroenterol Hepatol. 2010;7:251-64.

124. Ding $Y$, Yanagi $K$, Cheng $C$, et al. Interactions between gut microbiota and non-alcoholic liver disease: The role of microbiota-derived metabolites. Pharmacol Res. 2019;141:521-9.

125. Della Torre S. Non-alcoholic Fatty Liver Disease as a Canonical Example of Metabolic InflammatoryBased Liver Disease Showing a Sex-Specific Prevalence: Relevance of Estrogen Signaling. Front Endocrinol (Lausanne). 2020;11:572490.

126. Besse-Patin A, Léveillé M, Oropeza D, et al. Estrogen Signals Through Peroxisome ProliferatorActivated Receptor-y Coactivator 1 a to Reduce Oxidative Damage Associated With Diet-Induced Fatty Liver Disease. Gastroenterology. 2017;152:243-56.

127. Suzuki A, Abdelmalek MF. Nonalcoholic fatty liver disease in women. Womens Health (Lond). 2009;5:191-203.

128. Klair JS, Yang JD, Abdelmalek MF, et al. A longer duration of estrogen deficiency increases fibrosis risk among postmenopausal women with nonalcoholic fatty liver disease. Hepatology. 2016;64:8591.

\section{Figures}




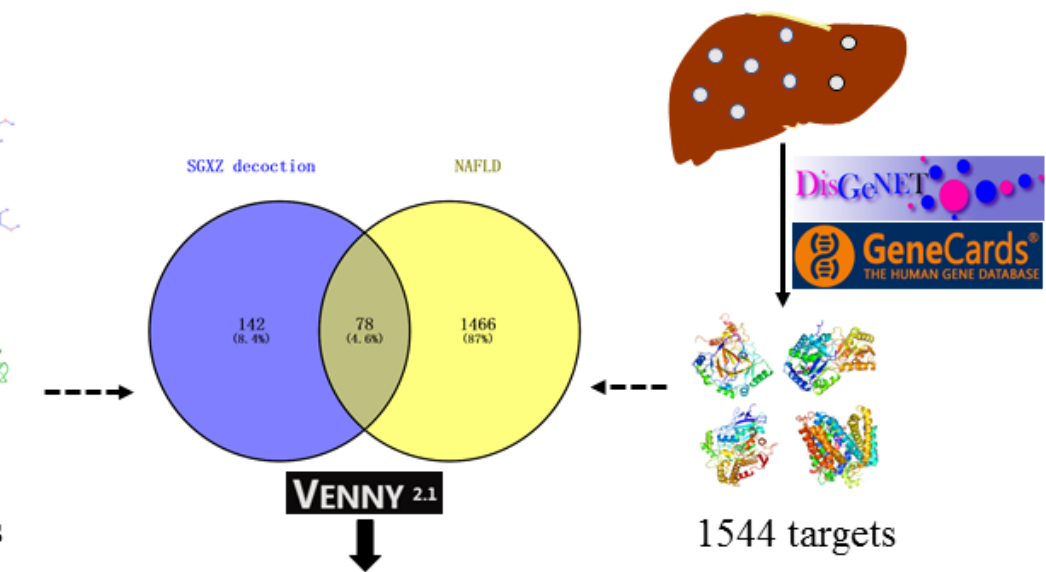

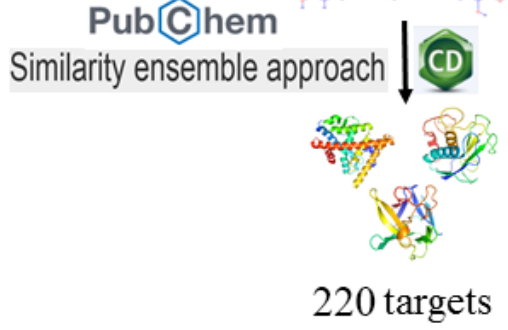

PubChem
Cytoscape
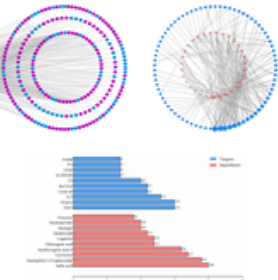

Ingredient-targēt-disease network

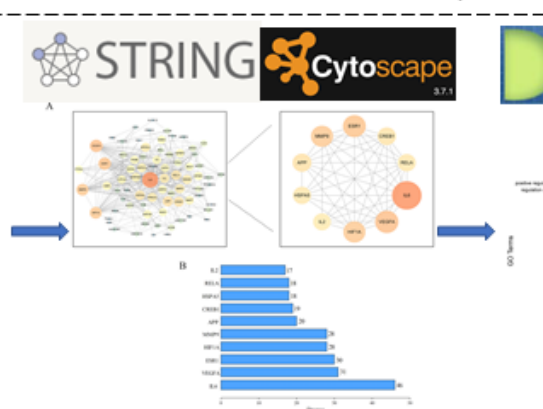

PPI network
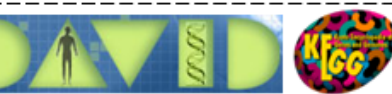

KEGG PATHWAY Database

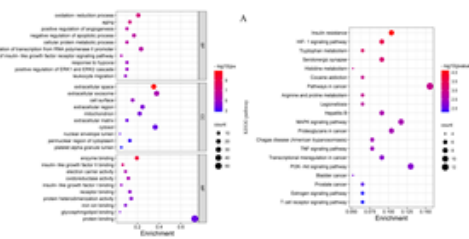

GO analysis

KEGG pathway analysis

\section{Figure 1}

Schematic diagram of the whole study design.

Figure 2 
2D structures of the 31 identified compounds in SGXZ decoction.

Figure 3

(A) Intersection targets between SGXZ decoction and NAFLD related targets via Venny diagram, (B) Compound-target network of SGXZ decoction. SGXZ decoction, chemical compounds and targets were colored as red, pink and purple respectively, and the 78 intersection targets in the network were displayed in blue, (C) Interaction network of compound-target-disease, the size of nodes corresponds to the value of the degree, (D) Top 10 putative compounds and targets with highest degree value in compound-targetdisease network.

A

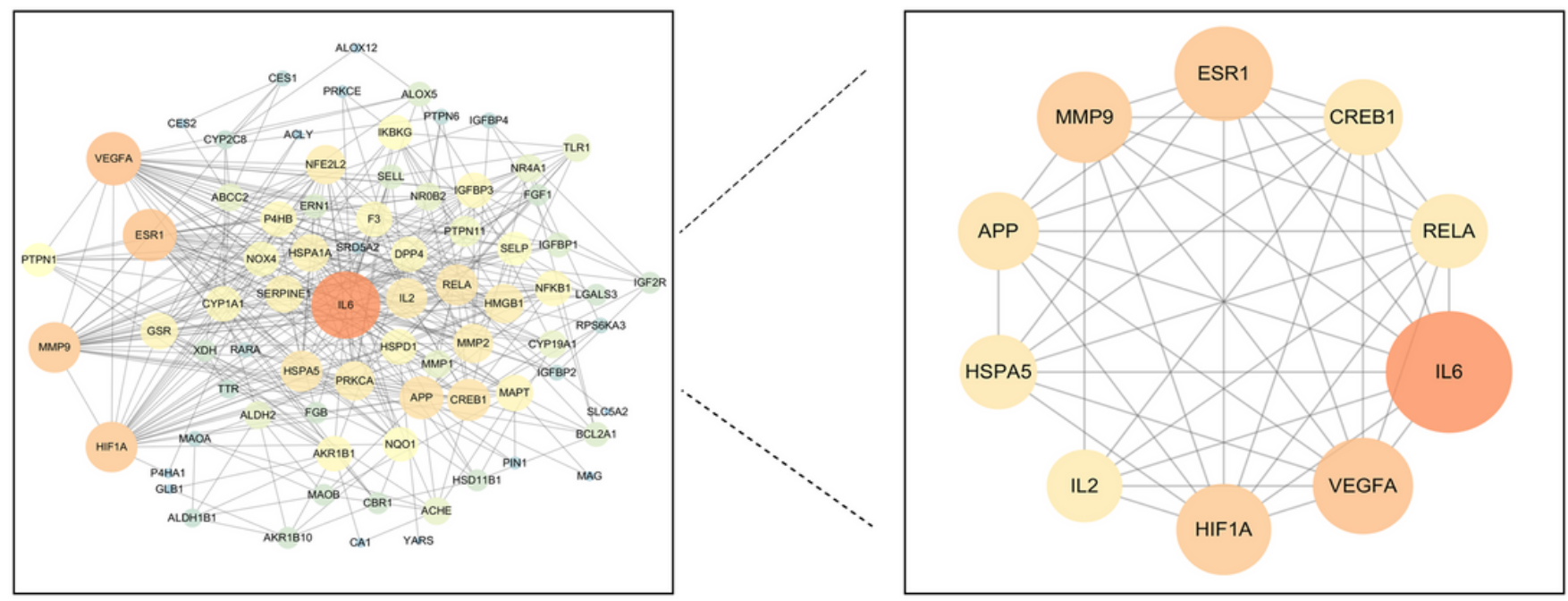

B

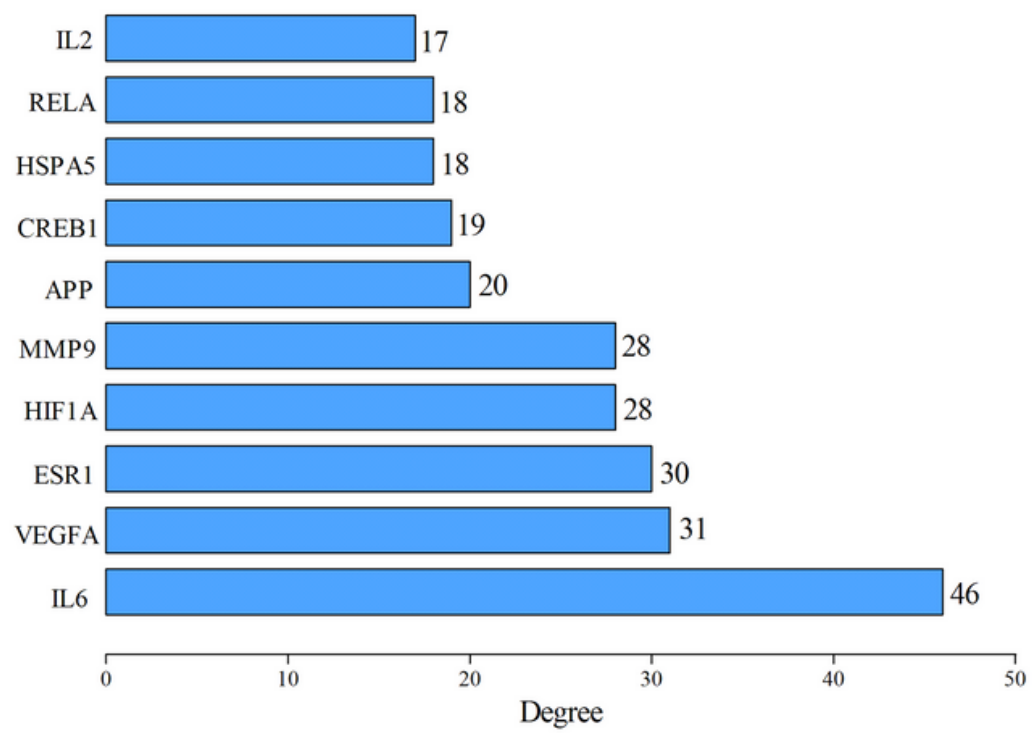

Figure 4 
(A) PPI network of potential targets of SGXZ decoction treating NAFLD. The size of the node with shade of the color indicated the corresponding degree value, (B) The identified top 10 putative target with degree value higher than the average according to the PPI network.

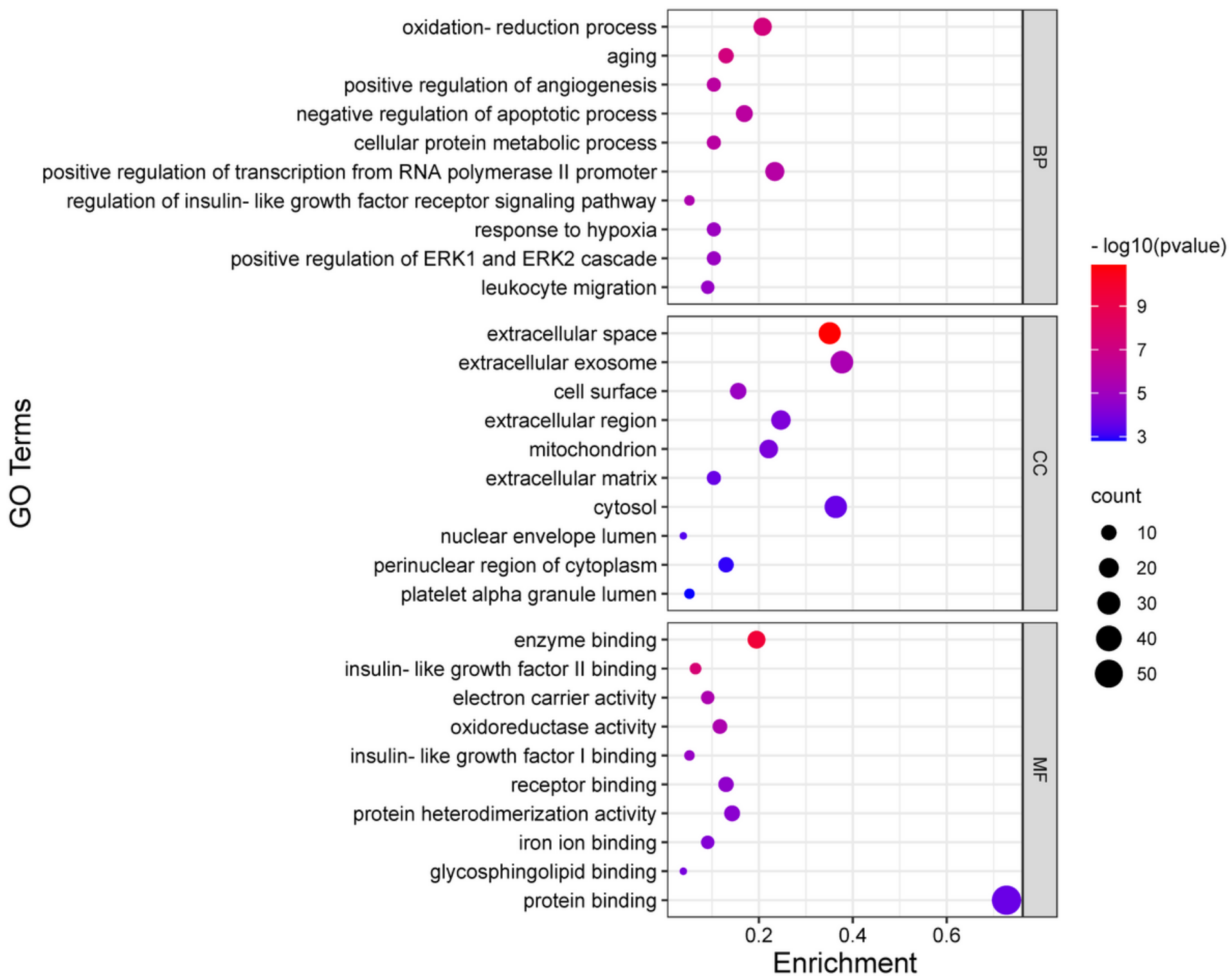

\section{Figure 5}

Representative $\mathbf{G O}$ enrichment analysis based on the targets in PPI network. Bubble plot of the top 10 terms of $\mathrm{BP}, \mathrm{CC}$ and MF. The bigger the dot, the more genes are enriched in the term, while the redder indicates the smaller $P$ value. 
A

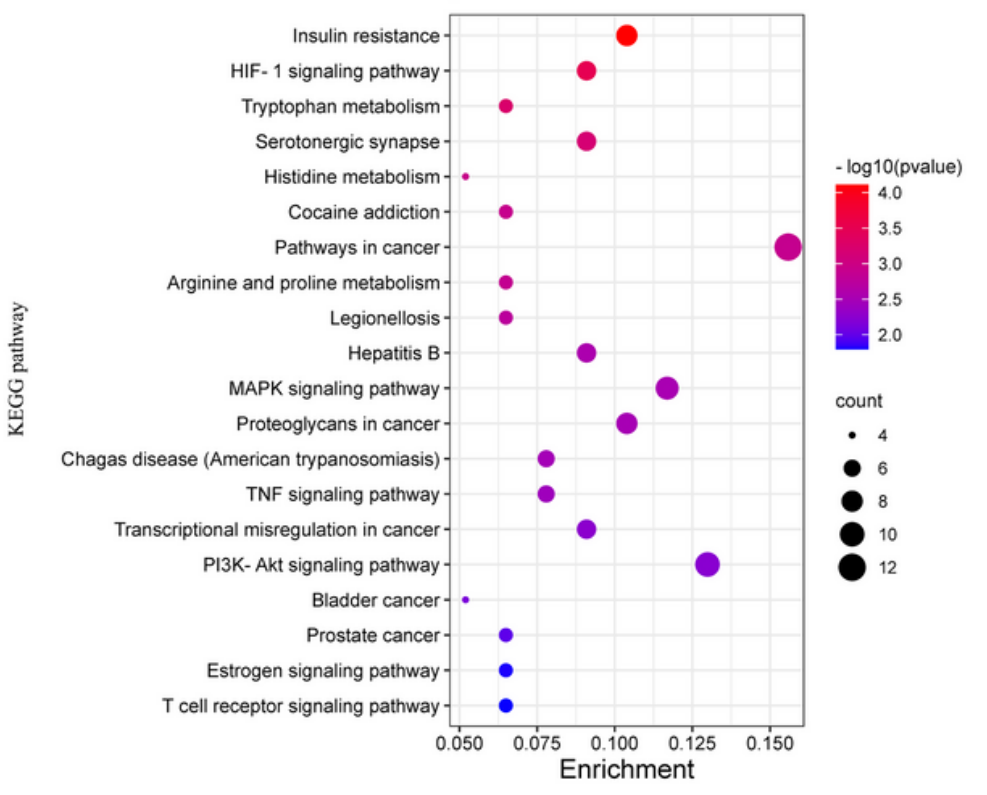

B

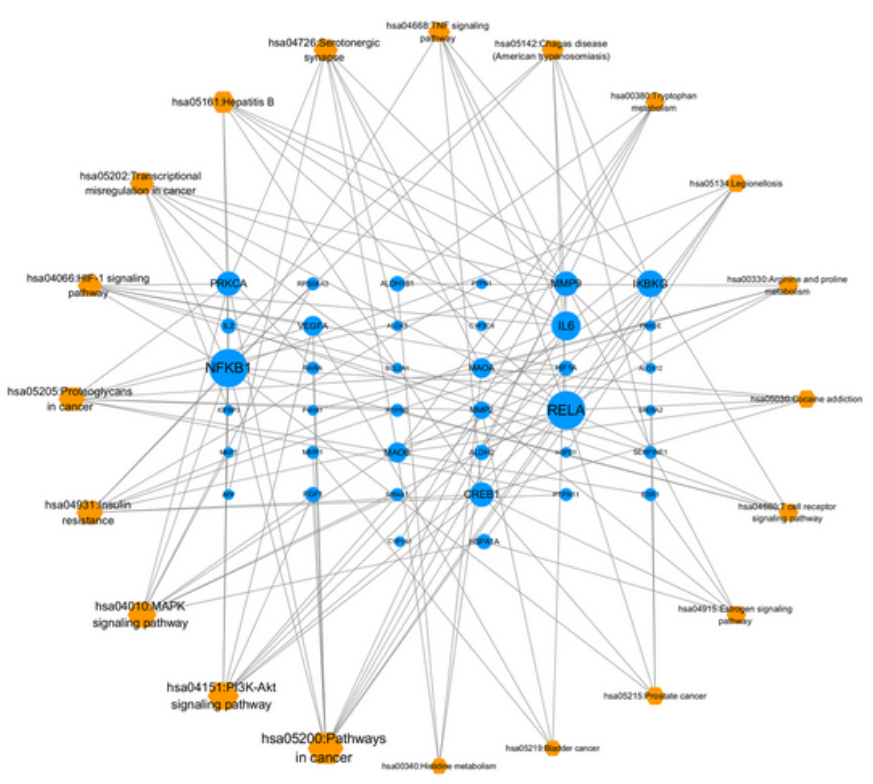

\section{Figure 6}

Results of KEGG pathway enrichment analysis based on the targets in PPI network. (A) The bubble plot of top 20 pathway enrichment analysis, (B) Target-pathway interaction network suggested the underlying mechanisms of SGXZ decoction for the treatment on NAFLD. Blue circle nodes indicated the targets and orange diamond represents the pathways. The gray edges linked the interaction between targets and pathways, and the node size was proportional to the degree value. 


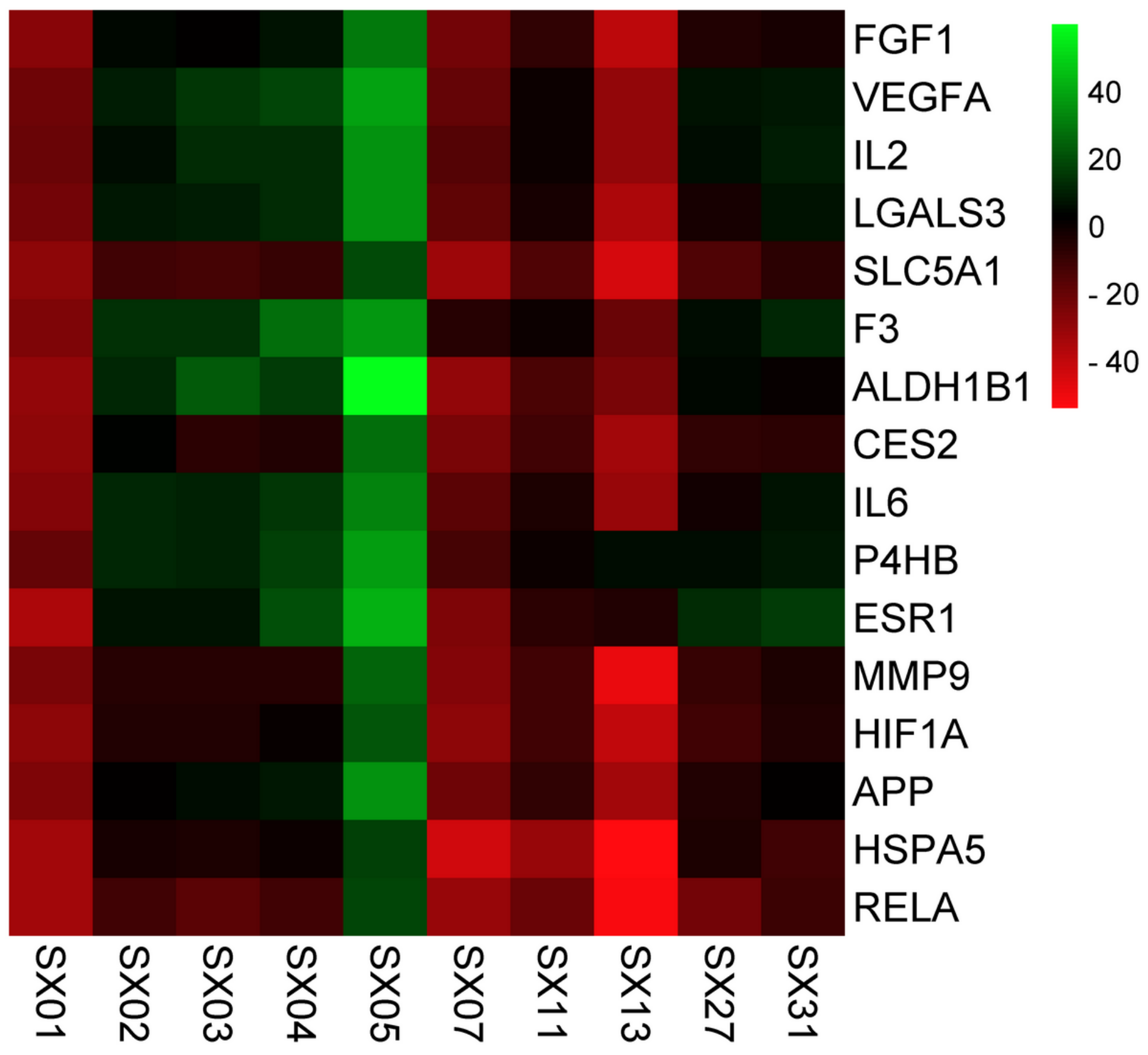

Figure 7

Heat map of the docking energy $(\mathrm{kcal} / \mathrm{mol})$ between key active ingredients and key targets. The redder the color, the lower the binding energy and the stronger the binding ability. 

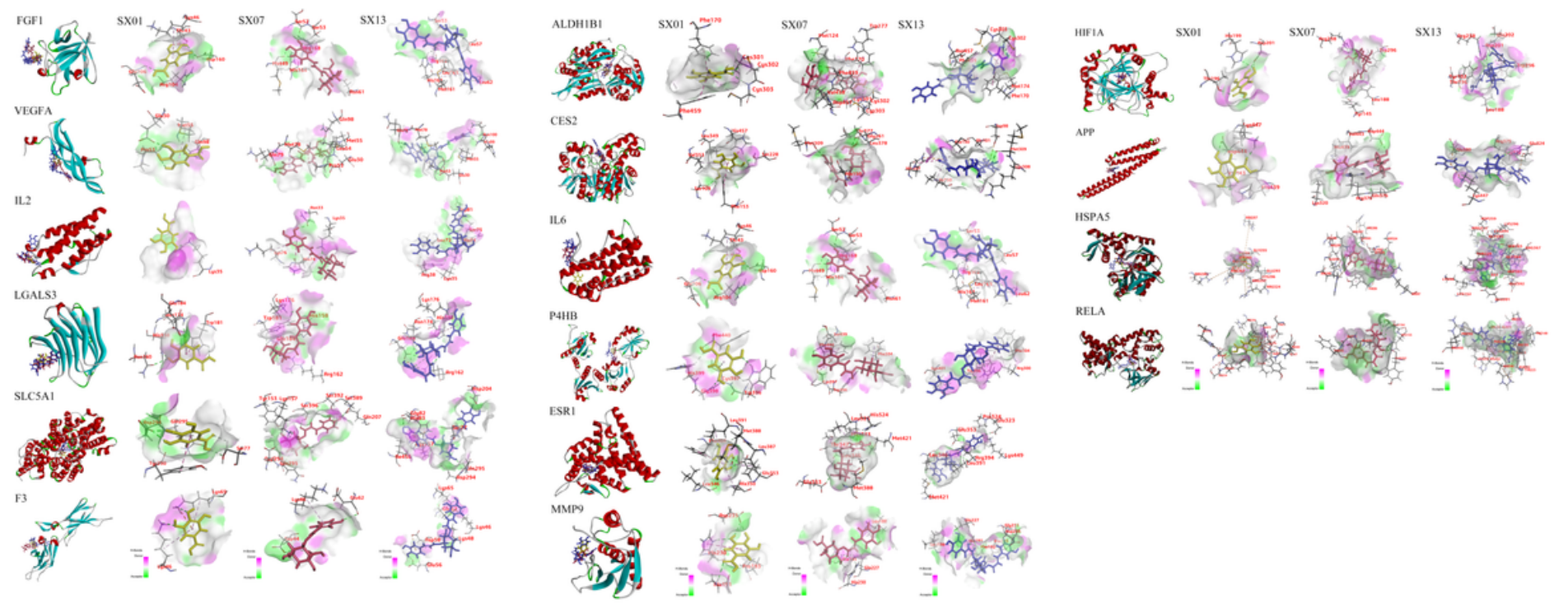

Figure 8

Interaction mode between SX01, SX07, SX13 and targets. Target proteins were displayed as solid ribbon and surface mode, and SX01, SX07 and SX13 were displayed in sticks and colored in yellow, pink and blue respectively. The residues interacting with the compounds were shown as sticks with their name labeled in red.

Figure 9

The predicted six core pathways and their interaction involved in the therapeutics mechanisms of SGXZ decoction treating NAFLD.

\section{Supplementary Files}

This is a list of supplementary files associated with this preprint. Click to download.

- SupplementaryFigure1.tif

- SupplementaryFile1.xlsx

- SupplementaryFile2.xlsx

- SupplementaryTable1.docx

- SupplementaryTable2.docx

- SupplementaryTable3.docx 